\title{
BMJ Open Differences in reimbursement listing of anticancer therapies in China: an observational study
}

\author{
Xiaodong Guan, ${ }^{1,2,3}$ Yichen Zhang (10 , ${ }^{1}$ Haishaerjiang Wushouer, ${ }^{4,5}$ \\ Luwen Shi (D) , ${ }^{1,3}$ Dennis Ross-Degnan, ${ }^{2}$ Anita Katharina Wagner ${ }^{2}$
}

To cite: Guan X, Zhang Y, Wushouer $\mathrm{H}$, et al. Differences in reimbursement listing of anticancer therapies in China: an observational study. BMJ Open 2020;10:e031203. doi:10.1136/ bmjopen-2019-031203

- Prepublication history and additional material for this paper are available online. To view these files, please visit the journal online (http://dx.doi. org/10.1136/bmjopen-2019031203).

Received 08 May 2019 Revised 31 October 2019 Accepted 21 November 2019

Check for updates

(c) Author(s) (or their employer(s)) 2020. Re-use permitted under CC BY-NC. No commercial re-use. See rights and permissions. Published by BMJ.

${ }^{1}$ Department of Pharmacy Administration and Clinical Pharmacy, School of Pharmaceutical Sciences, Peking University, Beijing, China ${ }^{2}$ Department of Population Medicine, Harvard Medical School and Harvard Pilgrim Health Care Institute, Boston, Massachusetts, USA

${ }^{3}$ International Research Center for Medicinal Administration,

Peking University, Beijing, China

${ }^{4}$ Center for Strategic

Studies, Chinese Academy of

Engineering, Beijing, China

${ }^{5}$ School of Medicine, Tsinghua University, Beijing, China

Correspondence to

Professor Luwen Shi; shiluwen211@163.com

\section{ABSTRACT}

Objective Access to highly priced anticancer medications usually requires insurance coverage. A first step towards coverage of such medications is their inclusion in reimbursement lists. We assessed listing for reimbursement in China between 2009 and 2018 of anticancer medications on the WHO's Essential Medicines List.

Setting and study design Using publicly available data, we assessed which anticancer medications listed in the 20th WHO Model List of Essential Medicines (EML) were included in China's National Reimbursement Drug List (NRDL). For five targeted anticancer medications on the WHO EML, we also assessed inclusion in the 31 Chinese Provincial Reimbursement Drug Lists (PRDLs). Logistic regression was used to test whether inclusion of targeted anticancer medications was associated with provincial economic levels.

Primary outcome measures Inclusion of five targeted anticancer medications in the NRDL and PRDLs before and after 2017.

Results The 2017 NRDL included all anticancer medications on the WHO EML (except for one not approved in China at the time), and by 2018, all 31 PRDLs listed the targeted anticancer medications except for nilotinib; four provinces had covered all five targeted medications before the 2017 NRDL coverage mandate. Provincial economic level and regional incidence of specific cancers seemed unrelated to the inclusion of five targeted anticancer medications in PRDLs.

Conclusion Our findings suggest that by including medications in the national and provincial reimbursement lists, China has taken an important first step in promoting access to targeted anticancer medications. Further research is needed to determine whether inclusion in PRDLs improved the availability, appropriate use and affordability of highly priced targeted anticancer medications in China.

\section{INTRODUCTION}

New anticancer medications have significantly changed the treatment of cancers in the last two decades. ${ }^{1}$ However, new therapies, especially targeted anticancer medications, are expensive and often unaffordable, limiting patients' options for cancer treatment. ${ }^{2} 3$ Aiming to satisfy the priority healthcare needs

\section{Strengths and limitations of this study}

- This study is the first to examine listing status on China's National Reimbursement Drug List (NRDL) and 31 Provincial Reimbursement Drug Lists (PRDLs) from 2009 to 2018 of 51 anticancer medicines listed on the WHO Essential Medicines List (EML) in 2017.

- Specifically, we assessed provincial listing for reimbursement of the five targeted anticancer medicines included in the 20th WHO EML.

- We also compared provincial listing status with provincial economic level and incidence of cancer types targeted by the five therapies.

- Listing for reimbursement is an important first step in expanding access to targeted anticancer medicines; future research should determine whether inclusion in PRDLs improved availability of or access to anticancer medications in China.

of the population, the WHO has proposed the concept of essential medicines and published its Model List of Essential Medicines (EML). ${ }^{4}$ The EML is a guide for governments to develop their own medicines lists to meet the needs of patients in their health systems; since insurance coverage is a prerequisite for access to highly priced anticancer medications, insurance reimbursement lists ideally include medicines deemed essential for quality cancer care.

Considering public health need, medication effectiveness and safety, the WHO has included several new, highly priced anticancer therapies in the EML. ${ }^{5}$ In 2017, the 20th version of WHO EML listed 51 anticancer medications, including 5 expensive targeted anticancer medications.

With increasing incidence and mortality, cancer has been the leading cause of death in China since $2010 .^{78}$ To promote access to care in general, including access to cancer care and anticancer medications, China has, since 2009, implemented Basic Medical Insurance (BMI).$^{9}$ The BMI, which by the end of 2018 had enrolled more than $94 \%$ of 
Chinese citizens, ${ }^{10}$ consists of three main health insurance schemes: the Urban Employee Basic Medical Insurance (UEBMI) for urban and retired employees, the Urban Resident Basic Medical Insurance (URBMI) for urban residents and the New Rural Cooperative Medical Scheme (NRCMS) for rural residents. ${ }^{11}$ Due to the widely differing coverage policies of provincial and municipal insurance systems in China, reimbursement rates differ widely by geographic area, system and population. Overall, the reimbursement rates of UEBMI are higher than URBMI rates, and the NRCMS has the lowest reimbursement rates. ${ }^{9}$ To provide better financial protection for the Chinese population, China's new medical reform in 2009 proposed to integrate URBMI and NRCMS into Urban and Rural Resident Medical Insurance (URRMI), ${ }^{12}$ although not all provinces had finished the integration by the end of $2018 .{ }^{9}$

The National Reimbursement Drug List (NRDL) is the guiding standard for BMI funds to pay for medications, and NRDL listing is an important step towards increasing access to expensive medications. ${ }^{13}$ Provinces are required to implement the most recent NRDL or issue their Provincial Reimbursement Drug List (PRDL) with limited modifications of the NRDL-provinces can delete, add or substitute up to $15 \%$ of the NRDL's medications ${ }^{14}$ to accommodate differences in economic development, insurance funding pools and reimbursement rates. Thus, reimbursement requirements for anticancer medications may differ across provinces and could lead to inequities in anticancer medication coverage. We assessed which anticancer medications listed on the 2017 WHO EML were included in the 2017 NRDL. Further, we analysed the variation in listing on PRDLs of five targeted anticancer medications across provinces in the last decade (2009-2018).

\section{METHOD}

We first compared the 2017 WHO EML with China's 2017 NRDL to assess overlap in listed anticancer medications. Then we selected the five targeted antineoplastic medications included in the 2017 WHO EML (trastuzumab, rituximab, imatinib, dasatinib and nilotinib) to investigate whether and when these medications were listed in the 31 PRDLs.

Data were collected from websites of the WHO,${ }^{15}$ the Ministry of Human Resources and Social Security of the People's Republic of China, ${ }^{14}{ }^{16}$ the State Medical Insurance Administration, ${ }^{17}$ the Center for Drug Evaluation of the National Medical Products Administration (NMPA), ${ }^{18}$ provincial Human Resources and Social Security Bureaus and provincial Health and Family Planning Commissions (see online supplementary appendix 1 ). We evaluated PRDLs in effect between 1 January 2009 and 31 October 2018. All data were separately checked by two researchers, and the inclusion dates in each provincial list of the five targeted medications confirmed in publicly available data.
We used logistic regression to test whether the inclusion of targeted anticancer medications in provincial lists was associated with provincial economic levels before 2017. The independent variable is the rank of 2017 provincial per capita Gross Domestic Product (GDP) provided by the China Health Statistical Yearbook 2018. ${ }^{10}$ Statistical analysis was performed using the statistical software Stata/MP V.14.0 (Revision 02 April 2015), StataCorp.

\section{Patient and public involvement}

No patients or members of the public were involved in this study.

\section{RESULTS}

\section{Listing of anticancer medications in the NRDL}

The 2017 NRDL was issued in February 2017 and updated following two rounds of government drug price negotiations in July 2017 and October 2018. Compared with the most recent 2009 NRDL, the 2017 and updated NRDL added 50 new anticancer medications and comprised 196 unique (by international non-proprietary name) anticancer medications in the following categories: traditional cytotoxic chemotherapy ( $\mathrm{n}=73$ unique international non-proprietary names), targeted anticancer medications $(\mathrm{n}=31$, details in online supplementary appendix 2), other antineoplastic medications $(n=49)$ and Chinese traditional medications (19 antineoplastic medications and 24 auxiliary antineoplastic medications). All but one (bendamustine, which had not been approved by the NMPA in 2017) of the 51 antineoplastic medications listed on the 2017 WHO EML were listed on the 2017 NRDL (see online supplementary appendix 3).

The five targeted anticancer medications added to the WHO EML in 2015 and 2017 were first approved by the NMPA between 2000 and 2011. All five medications were included in the 2017 NRDL and its updated versions (July 2017 and October 2018) following price negotiations (table 1).

\section{Listing of targeted anticancer medications on PRDLs}

By 31 October 2018, all PRDLs had been updated following publication of the 2017 NDRL, and all targeted anticancer medications except nilotinib were listed on 16 PRDLs (figure 1B). Twenty provinces had included at least one targeted anticancer medication on at least one insurance system's list before 2017 (figure 1A).

\section{Variation across provincial listing of targeted anticancer medications before 2017}

Table 2 illustrates the characteristics of provinces and the listing of targeted anticancer medications by different provincial insurance schemes. Before 2017, 11 provinces had no reimbursement lists listing any of the five medications, while four provinces covered all five medications (figure 1A). Interestingly, these provinces ranked low in per capita GDP. By contrast, none of the top three municipalities in terms of per capita GDP (Beijing, Shanghai 
Table 1 NMPA authorisation, WHO EML and NRDL listing of five targeted anticancer medications

\begin{tabular}{lllll}
\hline Targeted anticancer medications & $\begin{array}{l}\text { Year of WHO-EML } \\
\text { inclusion }\end{array}$ & $\begin{array}{l}\text { Year of NMPA } \\
\text { approval }\end{array}$ & $\begin{array}{l}\text { Year of NMPA generic } \\
\text { approval }\end{array}$ & $\begin{array}{l}\text { Year.months of } \\
\text { NRDL listing }\end{array}$ \\
\hline Rituximab & 2015 & 2000 & NA & 2017.7 \\
\hline Trastuzumab & 2015 & 2002 & NA & 2017.7 \\
Imatinib & 2015 & 2002 & 2013 & 2017.2 \\
Dasatinib & 2017 & 2011 & 2013 & 2017.2 \\
Nilotinib & 2017 & 2009 & NA & 2018.10 \\
\hline
\end{tabular}

EML, Model List of Essential Medicines; NMPA, National Medical Products Administration; NRDL, National Reimbursement Drug List.

and Tianjin) included any of the five targeted anticancer medications in their PRDLs before 2017. Inclusion of the five targeted medications was not statistically related to provincial per capita GDP rank ( $\mathrm{r}=0.05, \mathrm{p}=0.216)$.

Prior to 2017, listing decisions were not always aligned with cancer risk. In terms of disease burden and mortality, ${ }^{19}$ provinces in the Southwest region with the lowest incidence and mortality of breast cancer did not include trastuzumab in their reimbursement lists. In the Northeastern region (three provinces) with the highest incidence of breast cancer, only Liaoning Province included trastuzumab in its PRDL in 2016. Similarly, for lymphoma, two provinces (of three) in the Southern region with the highest lymphoma incidence and mortality included rituximab, while three provinces (of five) in the Northwest region with the lowest incidence also listed rituximab. In the Southern region with the highest incidence and mortality of leukaemia, only one province (of three) covered the three indicated tyrosine kinase inhibitors.

In terms of insurance type, most provinces began to incorporate targeted anticancer medications into their BMI or urban insurance system reimbursement lists after 2010. For example, 13 of 14 provinces listed rituximab in their BMI and 1/14 in the combined UEBMI and URBMI reimbursement list. Only four provinces included any targeted anticancer medications in their NRCMS reimbursement lists before 2017: Inner Mongolia Autonomous Region, Hebei, Fujian and Guizhou.

\section{DISCUSSION}

The national and provincial Chinese governments seek to provide safe, affordable and effective healthcare to all citizens by 2020 by increasing health insurance coverage and establishing a national essential drug programme. ${ }^{20}$ Our findings document that since the 2009 NRDL, the national and provincial governments have increasingly added anticancer medications, including new targeted anticancer medications, to their reimbursement lists.

We found that all 51 WHO EML-listed essential anticancer medicines (except for one that was not yet approved in China) were listed in the NRDL. Compared with previous studies of WHO-EML listed anticancer medications listed in national EMLs of 135 countries $^{21}$ and those of countries in South-East Asia, ${ }^{22}$ the number of anticancer medications listed on China's NRDL exceeds the world average. Listing for reimbursement is a prerequisite, but not sufficient, for expanding economically equitable access to these expensive treatments. ${ }^{23}$ Interestingly,

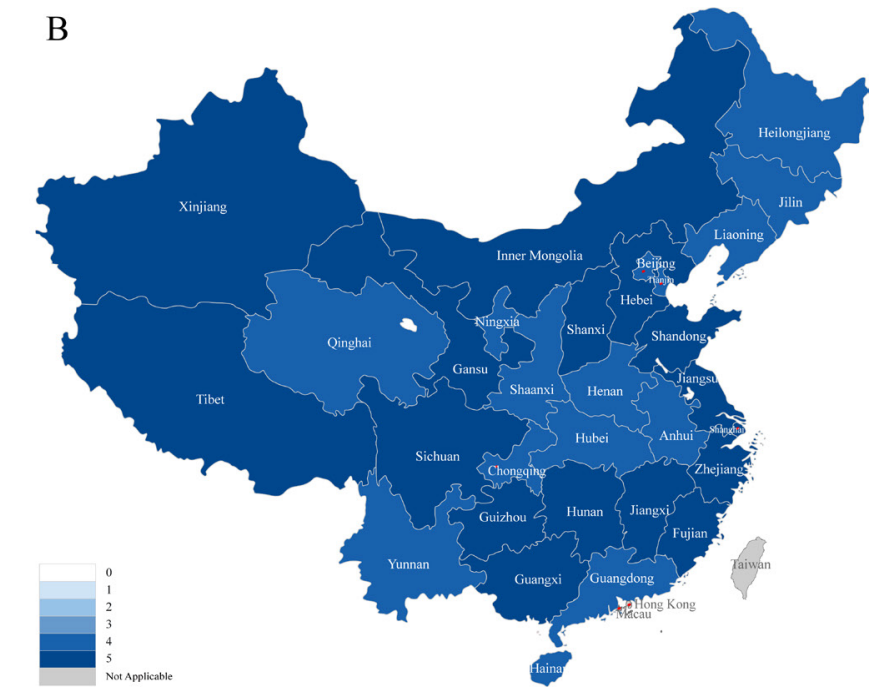

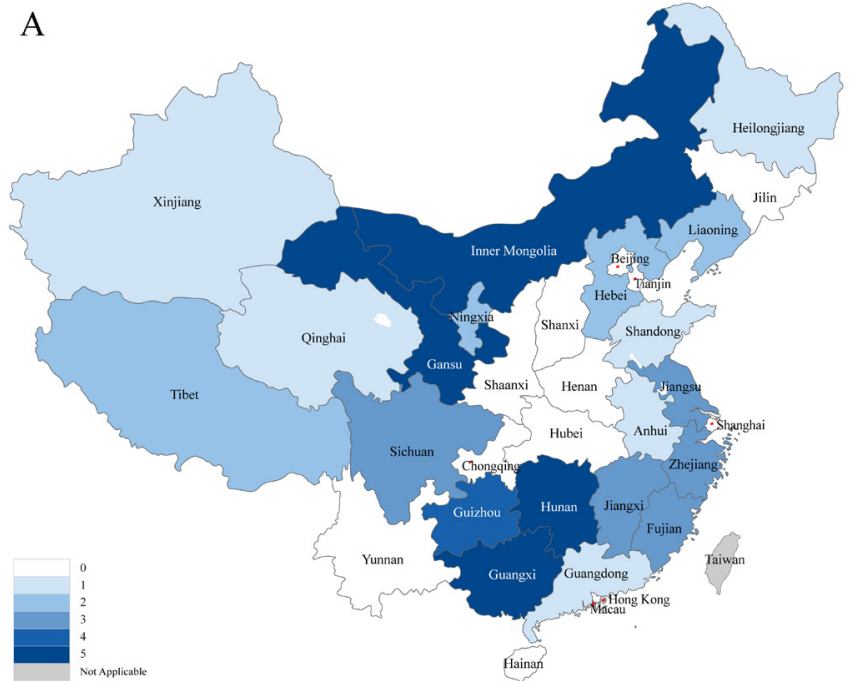

Figure 1 (A) Inclusion of five targeted anticancer medications in PRDLs of at least one insurance system before 2017. (B) Inclusion of five targeted anticancer medications in PRDLs after 2017. PRDL, Provincial Reimbursement Drug List. 


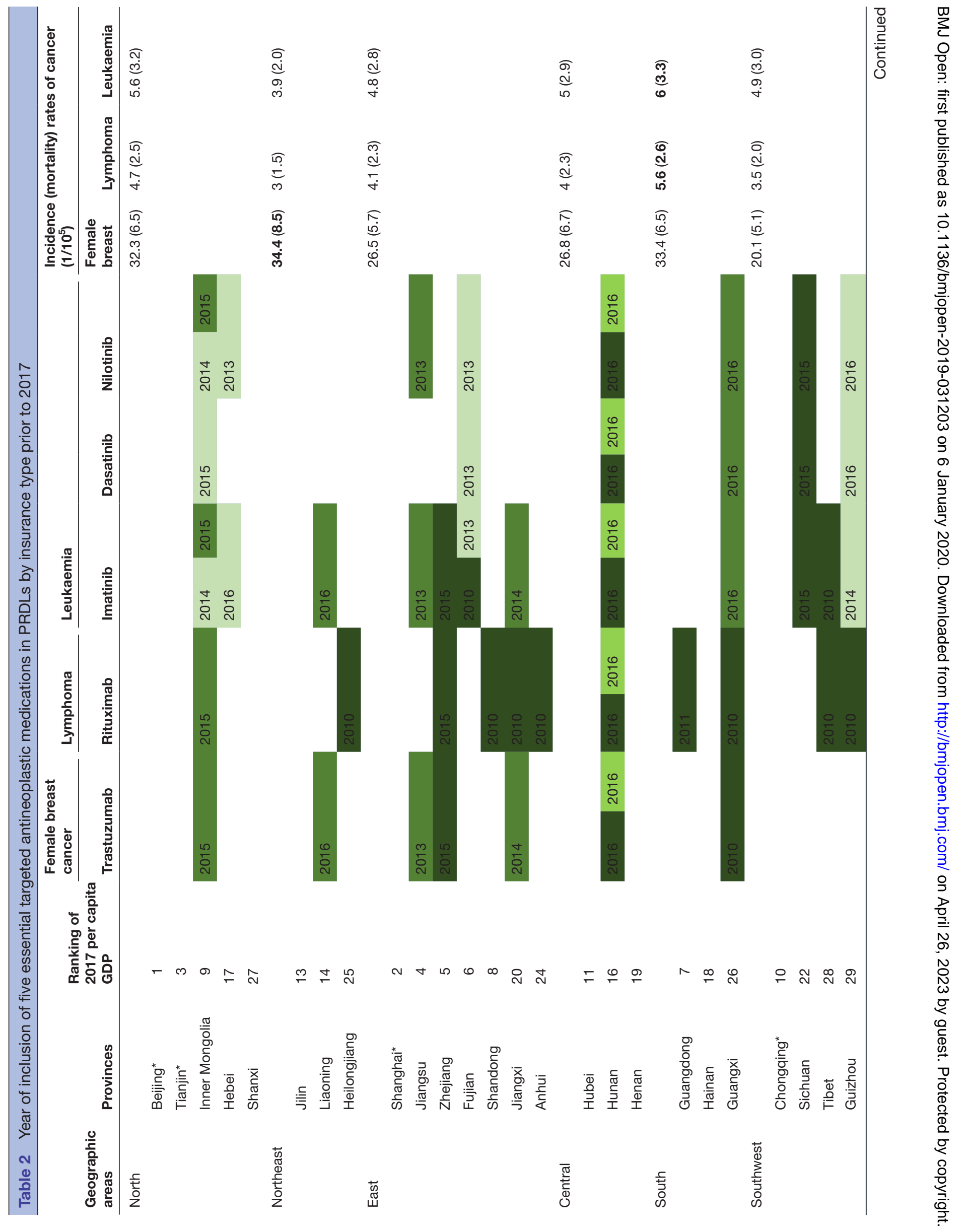




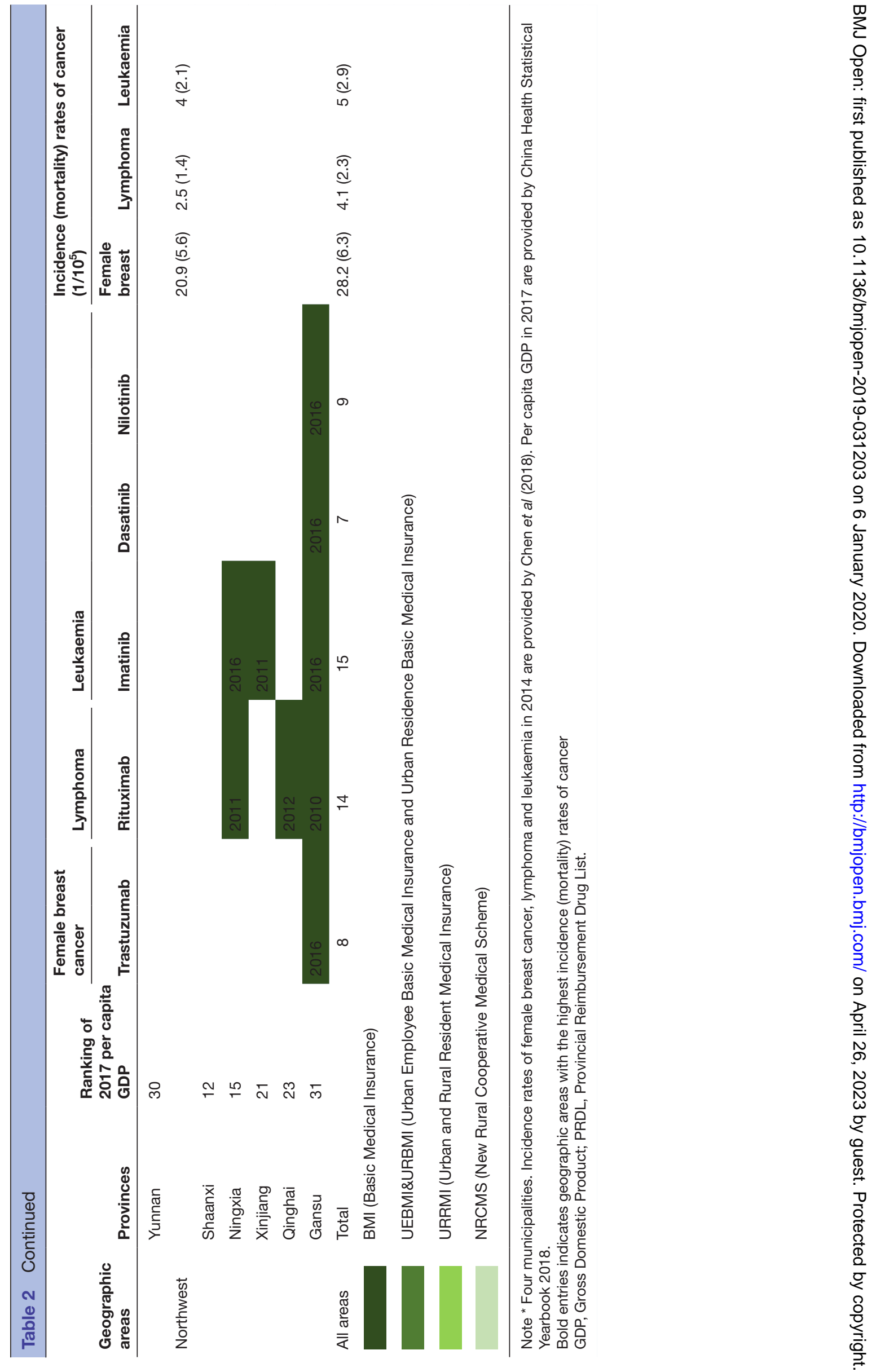


neither provincial wealth nor regional cancer morbidity appear to have been highly correlated with inclusion of targeted anticancer medications on PRDLs prior to 2017. Indeed, several economically disadvantaged provinces included all five expensive targeted medications while none of the three most economically developed municipalities incorporated any into their PRDLs.

There are two possible explanations for these observations. First, since implementation of insurance benefits is coordinated at the municipal level, provincial medical insurance listing does not signify a budgetary commitment for allocating funds; at the provincial level, the PRDL is a guiding document. At the municipal level, however, listing would require a commensurate budget allocation. That is, for economically developed municipalities (eg, Beijing, Shanghai, Tianjin), listing would require budget allocation and might result in unexpected budget impact. Second, in underdeveloped areas, due to the high outof-pocket (OOP) copayments, listed medications may be still unaffordable, even with insurance coverage. ${ }^{24} 25$ In underdeveloped areas, few patients can afford expensive targeted anticancer medications; therefore, expected impact on local medical insurance funds following listing for reimbursement could be limited. Similarly, due to the NRCMS's higher copayments compared with the UEBMI and URBMI, ${ }^{26}$ the costs of targeted medications would be too high for most rural villagers to afford, even with insurance coverage. ${ }^{24}{ }^{27}$ The true effect on affordable access of listing expensive medications on reimbursement lists is thus unknown, especially for NRCMS enrollees and those in underdeveloped provinces.

\section{LIMITATION OF THIS STUDY}

There are several limitations of our study. First, all information we used in this study was captured from government websites, and they might not provide complete information. However, two independent investigators separately collected and double checked the information, and the inclusion time of the five targeted medications were cross-checked against information in social media. Second, due to the limited availability of provincial cancer incidence and mortality data, we could not statistically analyse the relation between inclusion of targeted anticancer medication and provincial cancer incidence or mortality. Third, as the NRDL is only a guide for BMI funding, listing in the NRDL and PRDLs may not mean actual reimbursement. Further research will be needed to evaluate whether inclusion in PRDLs has improved access to the listed anticancer medications in China.

\section{CONCLUSION}

Our findings suggest that by including medications in the national and provincial reimbursement lists, China has taken an important first step in promoting access to targeted anticancer medications. Further research is needed to understand whether inclusion in reimbursement lists has promoted the availability, appropriate use and affordability of highly priced targeted anticancer medications.

Contributors XG, AKW, DR-D and LS conceptualised and designed the study. $Y Z$ screened and completed data extractions. YZ and HW contributed to analysis of the data. XG, AKW and DR-D conducted the final analysis and drafted the initial manuscript. All authors contributed to the critical revision of the paper and approved the final manuscript.

Funding This study was funded by National Natural Science Foundation of China (Grant No.71774005). The funders had no role in study design, data collection and analysis, decision to publish or preparation of the manuscript. Dr Wagner received partial support from the Department of Population Medicine Ebert Award.

Map disclaimer The depiction of boundaries on this map does not imply the expression of any opinion whatsoever on the part of BMJ (or any member of its group) concerning the legal status of any country, territory, jurisdiction or area or of its authorities. This map is provided without any warranty of any kind, either express or implied.

Competing interests None declared.

Patient consent for publication Not required.

Ethics approval The study was considered not human subjects research by the Harvard Pilgrim Health Care Institutional Review Board.

Provenance and peer review Not commissioned; externally peer reviewed.

Data availability statement Data are available in a public, open access repository. All data relevant to the study are included in the article or uploaded as supplementary information.

Open access This is an open access article distributed in accordance with the Creative Commons Attribution Non Commercial (CC BY-NC 4.0) license, which permits others to distribute, remix, adapt, build upon this work non-commercially, and license their derivative works on different terms, provided the original work is properly cited, appropriate credit is given, any changes made indicated, and the use is non-commercial. See: http://creativecommons.org/licenses/by-nc/4.0/.

\section{ORCID iDs}

Yichen Zhang http://orcid.org/0000-0001-7093-1044

Luwen Shi http://orcid.org/0000-0003-2683-6685

\section{REFERENCES}

1 National Cancer Institute. Targeted cancer therapies, 2019. Available: https://www.cancer.gov/about-cancer/treatment/types/targetedtherapies/targeted-therapies-fact-sheet [Accessed Apr 2019].

2 Carrera PM, Kantarjian HM, Blinder VS. The financial burden and distress of patients with cancer: understanding and stepping-up action on the financial toxicity of cancer treatment. CA Cancer J Clin 2018;68:153-65.

3 Shih Y-CT, Xu Y, Liu L, et al. Rising prices of targeted oral anticancer medications and associated financial burden on Medicare beneficiaries. J Clin Oncol 2017;35:2482-9.

4 World Health Organization. Essential medicines. Available: https:// www.who.int/topics/essential_medicines/en/ [Accessed Aug 2019].

5 Mayor S. Who includes 16 new cancer drugs on list of essential medicines. Lancet Oncol 2015;16:757.

6 World Health Organization. The selection and use of essential medicines. Report of the who expert Committee, 2015 (including the 19 th who model list of essential medicines and the 5 th who model list of essential medicines for children). Geneva 2015.

7 Chen W, Zheng R, Baade PD, et al. Cancer statistics in China, 2015. CA Cancer J Clin 2016;66:115-32.

8 Global, regional, and national age-sex specific all-cause and causespecific mortality for 240 causes of death, 1990-2013: a systematic analysis for the global burden of disease study 2013. The Lancet 2015;385:117-71.

9 Meng $\mathrm{Q}$, Fang $\mathrm{H}$, Liu X, et al. Consolidating the social health insurance schemes in China: towards an equitable and efficient health system. The Lancet 2015;386:1484-92.

10 National Health Commission of the People's Republic of China. China health statistical yearbook 2018. Beijing: China Union Medical University Press, 2018.

11 Sun Y, Gregersen H, Yuan W. Chinese health care system and clinica epidemiology. Clin Epidemiol 2017;9:167-78. 
12 State Council. Opinions of the State Council on Integrating the Basic Medical Insurance Systems for Urban and Rural Residents, 2016. Available: http://www.gov.cn/zhengce/content/2016-01/12/content_ 10582.htm [Accessed Mar 2019].

13 Wilson A, Cohen J. Patient access to new cancer drugs in the United States and Australia. Value Health 2011;14:944-52.

14 Ministry of Human Resources \& Social Security, 2017. Available: http://www.mohrss.gov.cn/SYrlzyhshbzb/shehuibaozhang/zcwj/ yiliao/201707/t20170718_274153.html [Accessed Mar 2019].

15 World Health Organization. WHO model list of essential medicines, 20th list, 2017. Available: https://www.who.int/medicines/ publications/essentialmedicines/en/ [Accessed Mar 2019]

16 Ministry of Human Resources \& Social Security. Notice of the Ministry of human resources and social security on Issuing the National drug catalog for basic medical insurance, work-related injury insurance, and maternity insurance (2017 edition), 2017. Available: http://www.mohrss.gov.cn/SYrlzyhshbzb/shehuibaozhang/ zcwj/yiliao/201702/t20170223_266775.html [Accessed Mar 2019].

17 State medical insurance administration, 2018. Available: http://www. gov.cn/xinwen/2018-10/10/content_5328891.htm [Accessed Mar 2019].

18 Center for drug evaluation of national medical products administration. Available: http://202.96.26.102/index/lists [Accessed Mar 2019].

19 Chen W, Sun K, Zheng R, et al. Cancer incidence and mortality in China, 2014. Chin J Cancer Res 2018;30:1-12.
20 Herd R, Y-W H, Koen V. Improving China's health care system 2010.

21 Robertson J, Barr R, Shulman LN, et al. Essential medicines for cancer: who recommendations and national priorities. Bull World Health Organ 2016;94:735-42.

22 Chivukula MV, Tisocki K. Essential cancer medicines in the National Lists of countries of the who south-east Asia region: a descriptive assessment. WHO South East Asia J Public Health 2018;7:90-8.

23 Bowman J, Rousseau A, Silk D, et al. Access to cancer drugs in Medicare Part D: formulary placement and beneficiary cost sharing in 2006. Health Aff 2006;25:1240-8.

24 Zhang A, Nikoloski Z, Mossialos E. Does health insurance reduce out-of-pocket expenditure? heterogeneity among China's middleaged and elderly. Soc Sci Med 2017;190:11-19.

25 Liu K, Wu Q, Liu J. Examining the association between social health insurance participation and patients' out-of-pocket payments in China: the role of institutional arrangement. Soc Sci Med 2014;113:95-103.

26 Su M, Zhou Z, Si Y, et al. Comparing the effects of China's three basic health insurance schemes on the equity of health-related quality of life: using the method of coarsened exact matching. Health Qual Life Outcomes 2018;16:41.

$27 \mathrm{Li} \mathrm{X}$, Zhang W. The impacts of health insurance on health care utilization among the older people in China. Soc Sci Med 2013;85:59-65 